\title{
The variation in lateral and longitudinal stress gauge response within an RTM 6 epoxy resin under one-dimensional shock loading
}

\author{
G.J. Appleby-Thomas ${ }^{1}$, P.J. Hazell and C. Stennett \\ Cranfield Defence and Security, Cranfield University, Shrivenham, Swindon, SN6 \\ $8 L A, U K$ \\ E-mail: g.applebythomas@cranfield.ac.uk
}

Tel. : +44 1793784192

\begin{abstract}
The dynamic response of a commercially important epoxy resin (RTM 6) has been studied using plate impact experiments in the impact velocity regime of $80-960 \mathrm{~m} / \mathrm{s}$. Both longitudinal and lateral manganin stress gauges were employed to study the development of orthogonal components of stress both during and after shock arrival. In light of recent work raising doubts about the interpretation of lateral gauge data, lateral response within the RTM 6 resin was also used to investigate the physical phenomena being measured by the embedded lateral gauges. $U_{S}-u_{P}$ and $\sigma_{X}-u_{P}$ Hugoniot relationships were in good agreement with data for similar polymer materials from the literature. Derivation of shear strength behaviour both during and after shock arrival showed evidence of strengthening behind the shock front, attributed to compression of the cross-linked epoxy resin polymer chains. Comparison of the change in lateral stress behind the shock to the behaviour of an epoxy resin possessing a similar $U_{S}-u_{P}$ Hugoniot from the literature showed a different response; likely attributable to enhanced cross-linking present in this second resin. This result suggests that the embedded lateral gauges were, at least in part, measuring a physical response behind the shock within the resin. A Hugoniot elastic limit of $0.88 \pm 0.04 \mathrm{GPa}$ was derived and found to be of the same order of magnitude as results found elsewhere for similar materials.
\end{abstract}

Keywords: Shock physics, RTM 6, lateral stress, shear strength, plate impact

\footnotetext{
${ }^{1}$ Corresponding author.
} 


\section{Introduction}

Polymer-based materials have found uses in applications ranging from explosive compositions and warhead design [1] to composite materials [2]. In many of these areas the final structure is routinely subject to severe shock during in-service life. Shock waves occur when the incident stress exceeds a materials dynamic flow strength; at this point, material shear strength (resistance to shear) is essentially zero and the material behaves in a fluid-like manner, e.g. hydrodynamically. The structure of certain materials, including many polymers, means that under such high strainrates changes in material properties occur. In such cases the material properties in question are said to be strain-rate dependant [3]. It is therefore of paramount importance to understand the response of polymer-based materials to high rates of strain (e.g. shock loading). Experimentally, the plate impact technique has been widely employed to investigate material strain rates $\geq 10^{6} / \mathrm{s}$ [4]. Compressive shocks are generated within a target material following impact of a flat/parallel flyer plate accelerated using a gun (typically driven by either compressed gas or a pyrotechnic charge). Inertial confinement results in a 1D state of strain being established within the target (maintained until release waves from the target edge catch up with the propagating shock). Suitable instrumentation (e.g. embedded stress gauges) then allows key experimental properties to be determined. These are: (a) $U_{S}$ - the propagation velocity of the induced shock through the target material; (b) $u_{P}$ - the continuum velocity of the material behind the shock front (known as the mass or particle velocity), and; (c) $\sigma_{X}$ - the longitudinal (Hugoniot) stress established within the target for the duration of the shock once the recording media has reached equilibrium. There are five key shock parameters required to describe materials behaviour; shock velocity, particle velocity, pressure, density and internal energy. However, only two of these parameters are required to uniquely define the material. A series of relations known as the Rankine-Hugoniot conservation equations, and based on conservation of mass, energy and momentum, allows the other shock parameters to be derived from the measured Hugoniot relationships. In this case, density and internal energy may be derived from either the $U_{S^{-}} u_{P}$ or $\sigma_{X}-u_{P}$ relationships. These relationships, known as Hugoniot equations-of-state, represent the $U_{S}, u_{P}, \sigma_{X}$ permutations a particular shocked material will physically pass through and, when combined with strength data, provide sufficient information to model the hydrodynamic response of the material in question [3].

Polymers fall into three broad groups [5]: thermoplastics - e.g. polymethymethacrylate (PMMA); themosets - e.g. epoxy resins, as considered here, and; elastomers such as polychloroprene (neoprene). Despite their important role in structural materials, only a limited body of work exists in the literature on the dynamic response of polymeric materials. Due to its use as a transparent window in interferometer-based particle velocity measurement systems, the behaviour of the thermoplastic PMMA has been extensively characterised by Barker and Hollenbach [6]. They observed a non-linear strain-rate dependence to its response at particle velocities/stresses below $0.7 \mathrm{~mm} / \mu \mathrm{s}$ and $22 \mathrm{kbar}$ respectively. This effect was linked to the presence of an elastic-plastic deformation mode - however, above this region a conventional linear $U_{S}-u_{P}$ response was noted. Millett and Bourne [7] have also conducted a detailed review of the shock response of 
Accepted for publication in the Journal of Materials Science in August 2009; subsequently published in Vol. 44, No. 22, pp. 6187-6198 (2009).

thermoplastic polymer materials - in this case focusing on the effects of side group size/complexity by considering polyethylene (H side groups), polypropylene (methyl side groups) and polystyrene (benzene ring side groups). Evidence of an increase in maximum shear strength with impact stress at elevated particle velocities/stresses was noted in all cases. This effect was observed to increase in magnitude with side group size/complexity, implying that the increasing scale of the side groups acted to progressively inhibit compression of the polymers. Elastomers have been suggested as potential constituents of composite armour systems - for example, Hazell et al. [8] conducted a series of ballistic tests involving penetration of a lead-antimony cored, $\mathrm{Cu}$ gilded, $7.62 \mathrm{~mm} \times 51 \mathrm{~mm}$ bullet into glass faced polyurethane replacement resin (PRR) targets. Understanding of the underlying penetration mechanisms was developed and a hydrocode material model derived (and calibrated against experiment) for the PRR. Both Millett et al. [5] and Bourne and Millett [9] investigated the shock response of the elastomer polchloroprene (neoprene) using the plate impact technique. They employed both longitudinal and lateral gauges to determine a Hugoniot and, in addition, investigate the change in lateral stress behind the shock. A divergence from the previously established linear Hugoniot (Marsh [10]) was found at lower particle velocities - in this case for $u_{P}<1.0 \mathrm{~mm} / \mu \mathrm{s}$. While this divergent section of the Hugoniot was taken to be linear, only four data points were employed. Consequently a non-linear response as observed at lower particle velocities with PMMA [6] may be an alternate explanation. An approximately constant gradient in lateral stress behind the shock at longitudinal impact stresses in the range 1.0 - 3.8 GPa was also observed (one shot at lower impact stresses did seems to imply a change in gradient, but this effect disappeared above $1.1 \mathrm{GPa}$ ). This result was taken to imply no change in strength of the material occurred in this impact stress regime.

Thermosetting polymers tend to exhibit a high degree of polymer chain cross-linking, imparting them with greater strength and temperature resistance over thermoplastic materials [11]. However, they are relatively brittle meaning that they are often reinforced with additions such as glass or carbon fibres, forming composite structures. The desirable physical and thermal properties of composite structures have led to them being employed in a wide variety of both armour and aerospace applications. For example, carbon fibre reinforced plastic (CFRP) has been widely employed in spacecraft structures due to the combination of its low density, high stiffness and low thermal expansion which leads to a high degree of thermal stability [2]. Thermosets such as epoxy resins (for example, Hexcel ${ }^{\circledR}$ RTM 6 [11]) have found a niche as matrix materials in such fibre reinforced structures. The dynamic response of carbon fibre composite systems with RTM 6 epoxy matrices has been investigated by a number of authors [1, 12]. However, carbon fibre reinforced systems are complex and have been shown to exhibit anisotropic responses dependant on fibre orientation. Consequently, it is important to understand the dynamic response of individual composite components. A number of different researchers have investigated the dynamic response of epoxy resins. Gerlach et al. [11] investigated strain rate effects in RTM 6, chosen for analysis due to its commercial importance as a matrix material in carbon fibre reinforced systems. They employed a range of experimental apparatus (including a tensile test 
machine, a hydraulic compression rig and a split-Hopkinson bar) to investigate the response of the resin at strain rates in the range $10^{-3}-10^{4} / \mathrm{s}$. A substantial strain rate dependence of yield stress, initial modulus and strain to failure was noted. In particular, the strain to failure in tension changed by $>50 \%$ over the range of strain rates investigated, emphasising the importance of understanding the dynamic properties of such resins. At higher strain rates, techniques such as plate impact experiments (described previously) are required. Munson and May [13] studied the response of three different forms of the epoxy resin Epon ${ }^{\mathrm{TM}} 828$ manufactured using different hardening agents/curing conditions. The different hardening agents used modified strongly the inherent cross-linking and therefore key material properties such as the glass transition temperature. While different responses were apparent at low strain rates, very similar Hugoniot relationships were found for all three variations (representing similar high strain rate responses). Hazell et al. [14] conducted a review of investigations into the dynamic response of epoxy resin systems and additionally independently derived a Hugoniot for RTM 6, providing data at higher strain rates than that of Gerlach et al. [11]. It was shown that in the case of five separate thermoset epoxy resin systems, resultant linear fits to experimentally derived $U_{S^{-}} u_{P}$ Hugoniot relationships were consistently very similar. Tentative evidence of an increase in strength with impact stress was observed. This conclusion was in good agreement with previous work by Millett et al. [15] where analysis of lateral gauge traces from a bespoke epoxy resin showed evidence of a negative gradient behind the shock whose magnitude increased with impact stress. This result was taken to be indicative of an increase in shear strength both with impact stress and behind the shock and was tentatively attributed to an inherent viscoelastic property of the epoxy in question.

Overall, it is apparent that there is relatively little information in the literature on the high strain rate behaviour of epoxy resins. In particular, relatively little work has been carried out on the lateral stress behaviour of such resins behind the shock. In this paper, the approach of Millett et al. [15] is extended to the commercially important resin transfer moulding (RTM) 6 resin, building on the description of its dynamic response set out by Hazell et al. [14]. Combinations of longitudinal and lateral gauges are used both to extend the previously derived RTM 6 Hugoniot and to investigate the behaviour of lateral stresses behind the shock. In addition, recent work by Winter and Harris [16] and Winter et al. [17] has suggested that perceived changes in strength behind the shock might, at least in part, be linked to the nature of lateral gauge mounting in targets, with shocks propagating at different velocities through the target/gauge packet system. This alternate explanation for observed phenomenon and its possible effects on the interpretation of the response of lateral gauges has not been discussed in light of epoxy resin systems. Consequently, this alternate explanation for the response of lateral gauges is taken into account in the context of lateral gauge results presented here in order to provide a broader scope for interpreting both this work and that conducted elsewhere. 


\section{Material properties}

Experimental work has focused on a commercially important epoxy/amine resin system developed by Hexcel Composites (Duxford UK) primarily for use in the advanced RTM process [18,19] and known by the trade name HexFlow ${ }^{\circledR}$ RTM 6. This resin is supplied in mono-component form; e.g. the epoxy and aromatic-amine curing elements are both present in the supplied mixture. The mono-component resin is cast at $80^{\circ} \mathrm{C}$, before an initial cure in the mould at $160^{\circ} \mathrm{C}$ for around 75 minutes and a final post-cure at $180^{\circ} \mathrm{C}$ for 2 hours, with temperature changes kept to $1^{\circ} \mathrm{C} /$ minute at each stage [20]. While the mono-component nature of the resin necessitates careful storage (unlike conventional two-component resins, mono-component systems can undergo spontaneous cure at elevated temperatures), the presence of just one element simplifies injection into the mould during the RTM process. The majority of epoxy resin systems are based on the reaction products of Bisphenol A (2,2'-Bis(4'-Hydroxyphenyl)Propane) with Epichlorohydrin (1-Chloro-2,3-EpoxyPropane), which react to form a polymer chain terminated by reactive epoxy groups; subsequently a curing agent reacts with the epoxy groups to form cross-links within the final resin [21]. Possible curing agents include aliphatic and aromatic amines (employed in RTM 6) as well as anhydrides. Final epoxy resin properties depend on numerous factors including: the choice of epoxy, curing agent and (where appropriate) plasticiser, and; the thermal profile of the cure. Broadly speaking slower cure rates and larger polymer chains lead to greater degrees of crosslinking and, therefore, greater stiffness. However, the complexity of this process makes it difficult to relate structure to final epoxy properties [21].

The main components of RTM 6 are: (1) an epoxy, Tetraglycidyl Methylene Dianiline (TGMDA), and; (2) two curing agents: (i) 4,4'-Methylenebis(2,6-Diethylaniline), and (ii) 4,4'Methylenebis(2-Isopropyl-6-Methylaniline) [22]. These three components are shown schematically in Fig. 1(a). For comparison, a short-chain version of Digilycidyl Ether (based on the reaction of Bisphenol A with Epichlorohydrin) is shown in Fig. 1(b); it is of note that this molecule exhibits a lower number of active O-based epoxy groups then in the case of TGMDA. Both of the curing agents employed are polyfunctional aromatic amines, with two reactive amine groups each. For commercial reasons, little information on the composition of RTM 6 is given in Ref. [22], beyond the likely proportions in the final epoxy resin of all three elements, namely: 30$60 \%$ epoxy (TGMDA), and; 10-40\% of each curing agent. Further, no information is available in the open literature on any plasticisers employed in this system. It has been shown previously that changes in structure (e.g. enlarged side groups [7]) can modify the shock response of polymers. Consequently, it seems reasonable to assume that the presence of different epoxy/curing system elements in RTM 6 will lead to a different shock response, particularly in terms of any strengthening behaviour, to other previously studied epoxy resin systems. 
Accepted for publication in the Journal of Materials Science in August 2009; subsequently published in Vol. 44, No. 22, pp. 6187-6198 (2009).

(a)

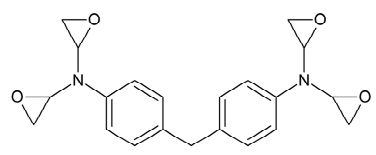

Tetraglycidyl Methylene Dianiline (TGMDA)

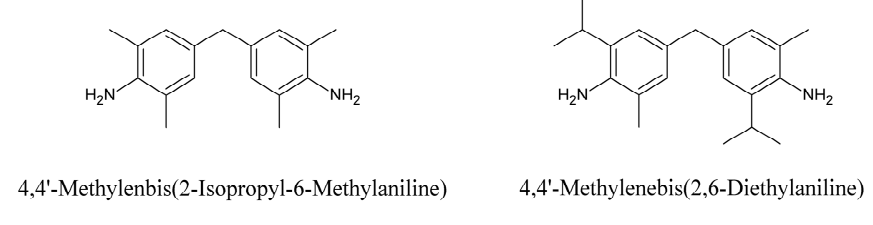

(b)

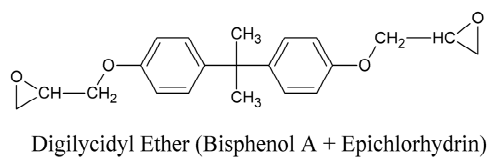

Fig. 1 (a) Chemical structure of key constituents of Hexcel ${ }^{\circledR}$ RTM 6 [22]; (b) Chemical structure of the single-repeating-element polymer created by reacting Bisphenol A with Epichlorohydrin [21].

In previous work on cured RTM 6, the authors of Ref. [14] used a Panametrics 5077PR pulse receiver in the pulse-echo configuration to measure longitudinal and shear wave velocities in cured RTM 6 resin. In combination with the density (measured using a Micrometrics AccuPyc 1330 gas pycnometer), these wave velocities were subsequently used to calculate the elastic properties of RTM 6 shown in Table 1.

Table 1 Elastic properties of cured RTM 6 resin.

\begin{tabular}{cccccc}
\hline Material & $\rho_{0}\left(\mathrm{~g} / \mathrm{cm}^{3}\right)$ & $c_{L}(\mathrm{~mm} / \mu \mathrm{s})$ & $c_{S}(\mathrm{~mm} / \mu \mathrm{s})$ & $c_{B}(\mathrm{~mm} / \mu \mathrm{s})$ & $v$ \\
\hline RTM 6 & $1.141 \pm 0.001$ & 2.699 & 1.284 & 2.256 & 0.35 \\
\hline
\end{tabular}

\section{Experimental technique}

Plate impact experiments were employed to investigate the dynamic response of RTM 6 to one dimensional loading under a variety of impact conditions. A $50 \mathrm{~mm}$ bore, $5 \mathrm{~m}$ barrel, single stage gas gun was used for all experiments [23]. Figs. 1(a) and (b) schematically illustrate: (a) a typical plate impact experimental setup where a lateral gauge was employed, and; (b) an exploded target showing the position of the embedded lateral gauges (type J2M-SS-580SF-025, manufactured by Vishay Micro-Measurements) relative to the target front face. 


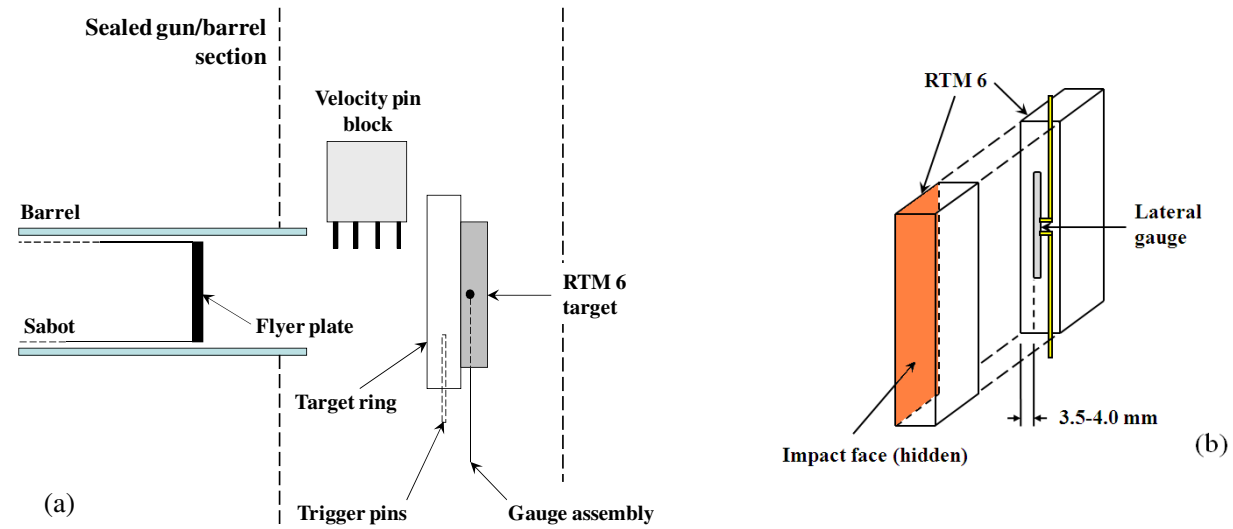

Fig. 2 (a) schematic illustration of a typical plate impact experimental setup; (b) schematic illustration (exploded) of a target containing an embedded lateral gauge.

Lateral targets, as shown in Fig. 2(b), were prepared via several stages: (i) first the impact surface of a block of RTM 6 was machined to a surface roughness of $<5 \mu \mathrm{m}$; (ii) secondly, the RTM 6 block was sectioned as shown in Fig. 2(b); (iii) thirdly, a lateral gauge, encapsulated in $25 \mu \mathrm{m}$ of Mylar for insulation/protection, was introduced 3.5-4.0 $\mathrm{mm}$ (measured) from the impact face and glued to one half of the RTM 6 block using a slow cure epoxy (Loctite 0151 HYSOL ${ }^{\circledR}$ EpoxiPatch $^{\circledR}$ Adhesive), before; (iv) the other half of the RTM 6 block was glued to the combined RTM 6 section/gauge package and the entire system clamped using a specially machined jig for a minimum of 24 hours until the slow cure epoxy set. The target ring/RTM 6 target assembly shown in Fig. 2(a) were arranged so that the entirety of the projectiles surface contacted the target simultaneously. Inertial confinement meant that strain was confined to the impact axis, with orthogonal elements reduced to zero - resulting in a planar compressive wave entering the target and traversing to the embedded lateral gauge. Lateral gauge interpretation was based on a modified form of the impedance matching technique. Developed by Rosenburg and Partom [24], this approach assumed that the strain in a thin (foil) laterally embedded gauge was equivalent to that of the surrounding material. The approach employed in converting measured changes in voltage across the manganin gauge to lateral stresses also considered both the elastic-plastic response of the manganin gauge [25] used as well as the pressure dependence of its response at stresses below its elastic limit [26]. In two cases longitudinal gauges (type LM-SS-125CH-048, manufactured by Vishay Micro-Measurements \& SR-4) were employed either in front or behind the target in addition to the lateral gauge. Further, one experiment was conducted in which only longitudinal gauges were employed. Where rear surface longitudinal gauges were employed a backing plate was used to provide support for the gauge. While an RTM 6 backing plate would have provided the optimum impedance match, due to limited supplies all material was reserved for use as targets; instead, a 12mm thick block of PMMA was employed in this role. The arrangement for the longitudinal gauge only case is illustrated in Fig. 3(a), while that for a lateral/rear gauge 
configuration is shown in Fig. 3(b). When three gauges were employed, an additional front surface gauge with the configuration shown in Fig. 3(a) was included ahead of the arrangement shown in Fig. 3(b).

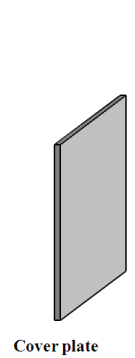

(a)

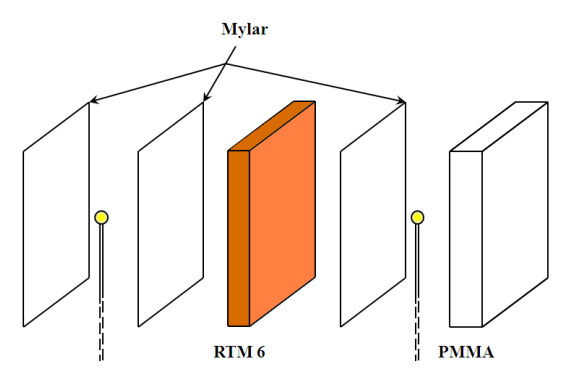

Gauge 1

Gauge 2

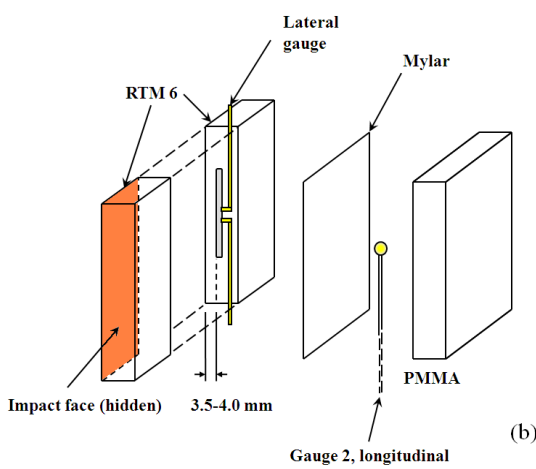

Fig. 3 (a) schematic illustration (exploded) of an RTM 6 target enclosed within two longitudinal gauge packages; (b) schematic illustration (exploded) of a target containing an embedded lateral gauge backed by a single longitudinal gauge.

\section{Results and discussion}

A total of four experiments were conducted incorporating embedded lateral gauges according to the arrangement shown in Fig. 2(a). These involved flyer plates of either PMMA or Cu impacting RTM 6 targets at velocities ranging from 193 to 960 m/s. Additionally, one test with longitudinal gauges only was carried out at an impact velocity of $80 \mathrm{~m} / \mathrm{s}$ according to the arrangement shown in Fig. 3(a). Table 2 summaries the experimental conditions employed in these tests. Lateral stresses are based on an average taken across the duration of the shock. $U_{S^{-}} u_{P}$ values and measured longitudinal stresses are only included for the three tests where front/rear surface gauges were employed. For the other two tests only estimated values of $\sigma_{X}$ (indicated) based on the Hugoniot for RTM 6 presented previously by Hazell et al. [14] are included. 
Accepted for publication in the Journal of Materials Science in August 2009; subsequently published in Vol. 44, No. 22, pp. 6187-6198 (2009).

Table 2 Summary of experimental results.

\begin{tabular}{|c|c|c|c|c|c|c|}
\hline$V_{\text {impact }}(\mathrm{m} / \mathrm{s})$ & $\begin{array}{c}\text { Flyer } \\
\text { material }\end{array}$ & $\begin{array}{c}\text { Flyer } \\
\text { thickness } \\
(\mathrm{mm})\end{array}$ & $\sigma_{Y}(\mathrm{GPa})$ & $\sigma_{X}(\mathrm{GPa})$ & $U_{S}(\mathrm{~mm} / \mu \mathrm{s})$ & $u_{P}(\mathrm{~mm} / \mu \mathrm{s})$ \\
\hline 80 & PMMA & 1.5 & ---- & 0.11 & 2.70 & 0.043 \\
\hline 193 & $\mathrm{Cu}$ & 10 & 0.23 & $\begin{array}{c}0.59 \\
\text { (estimated) }\end{array}$ & ---- & ---- \\
\hline 472 & $\mathrm{Cu}$ & 5 & 1.05 & 1.69 & 3.45 & 0.426 \\
\hline 665 & $\mathrm{Cu}$ & 10 & 1.69 & $\begin{array}{c}2.44 \\
\text { (estimated) }\end{array}$ & ---- & ---- \\
\hline 960 & $\mathrm{Cu}$ & 5 & 3.05 & 4.44 & 3.97 & 0.854 \\
\hline
\end{tabular}

Typical traces from the $960 \mathrm{~m} / \mathrm{s}$ shot which featured both front and rear longitudinal gauges in addition to a centrally located lateral gauge are shown in Fig. 4. Raw data is presented with no processing beyond conversion of recorded voltages to stresses and rescaling of the time base in order to enhance clarity (the starting point for all three traces has been offset by the same amount in order to position the initial rise of the front surface gauge at ca.0.5 $\mu \mathrm{s}$ ).

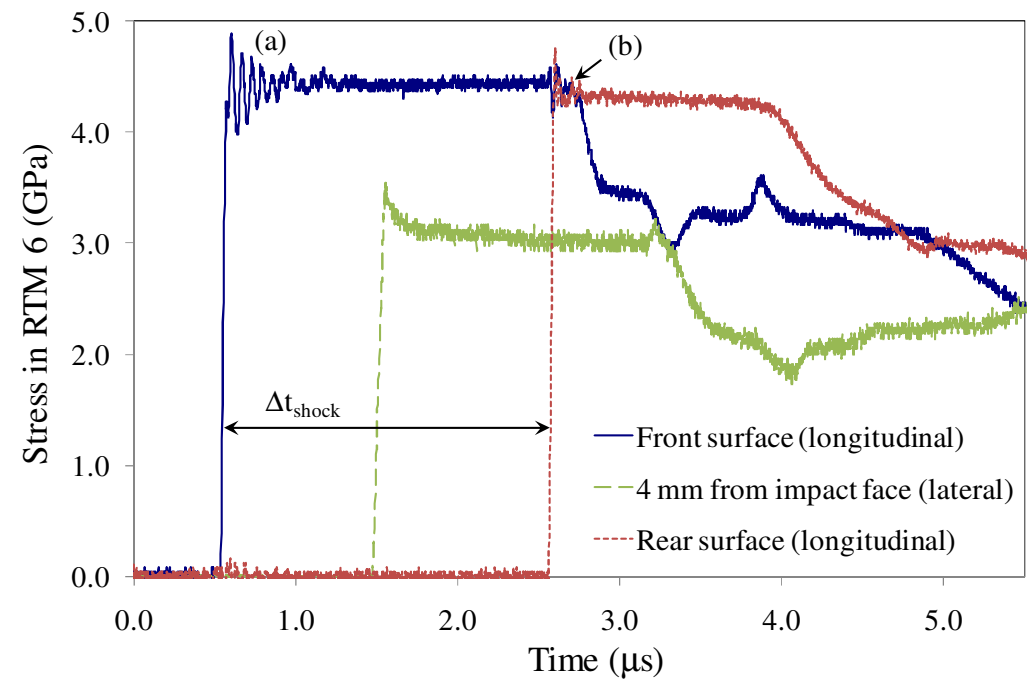

Fig. 4 Front and rear longitudinal gauge traces (stress in RTM 6), plus a central lateral gauge trace, generated following impact of a $5 \mathrm{~mm}$ thick $\mathrm{Cu}$ flyer on an $8 \mathrm{~mm}$ thick RTM 6 target at 960 $\mathrm{m} / \mathrm{s}$. 
An arrow showing the time interval $\Delta \mathrm{t}_{\text {shock }}$ is included in Fig. 4. This represents the duration between shock arrival at the front and rear longitudinal gauges. Given known target/encapsulating mylar thickness, this allowed calculation of the shock velocity. The rear surface trace in Fig. 4 has been rescaled to represent the stress in the RTM 6 resin rather than the PMMA backing using Eq. 1.

$$
\sigma_{R T M 6}=\frac{1}{2} \frac{\left(Z_{R T M 6}+Z_{P M M A}\right)}{Z_{P M M A}} \sigma_{P M M A}
$$

where $\sigma_{R T M} 6$ is the stress in the RTM 6, $\sigma_{P M M A}$ is the stress in the PMMA, $Z_{R T M} 6$ the impedance of the RTM 6 and $Z_{P M M A}$ the impedance of the PMMA. In general, $Z=\rho_{0} U_{s}$, where $\rho_{0}$ is the material density and $U_{s}$ is the shock velocity.

The slight difference in magnitude of the resultant Hugoniot stresses in the front/back surface longitudinal gauges in Fig. 4 is attributed to experimental errors arising from issues such as the presence of epoxy layers of unknown thickness (typically $<100 \mu \mathrm{m}$ ). These errors led to uncertainty in the calculated shock - and therefore particle - velocities which, as the impedance of both materials depends on the calculated shock/particle velocities, led to slight errors in the application of Eq. 1. Nonetheless, and despite the fact that Eq. 1 is primarily designed for use with hydrodynamic (fluid) systems, as the difference between the resultant Hugoniot stresses was just $0.14 \mathrm{GPa}$ - equivalent to just $3.2 \%$ of the stress quoted in Table 2, the application of Eq. 1 to the comparison of stresses in RTM 6 resin/PMMA appears justified. Noise evident at points (a) and (b) in Fig. 4 has been observed on such manganin gauge traces previously and appeared electrical in nature (e.g. the fast rise time of the shock leads to electrical ringing within the gauge) [14, 27]. The fact that a similar oscillation to that in the rear surface longitudinal gauge at point (b) is apparent on the front surface gauge immediately behind this trace appears to confirm the supposition that some form of electrical interference is responsible for these features of the two traces. Rise times for both types of gauge were relatively fast - for the longitudinal gauges they were typically ca.50 ns, whereas the lateral gauge responded slightly more slowly rising to a plateau after ca.75 ns. The $U_{S}-u_{P}$ data points from Table 2 are plotted against the Hugoniot for RTM 6 resin previously derived by Hazell et al. [14] in Fig. 5. Errors in shock velocity (and therefore subsequently calculated particle velocity) were based on the earliest/latest possible arrival times at each gauge according to the approach set out in Fig. 4. For the purpose of comparison, Hugoniot relationships for two different epoxy resins studied by Munson and May [13] and Millett et al. [15] are also included. These two epoxy resins have different compositions to RTM 6. In the case of Munson and May [13], the epoxy considered here was Epon 828 combined with a hardener known as agent Z (see Ref. [13] for further details). Epon 828 is based 
on the reaction between Bisphenol A and Epichlorohydrin, with the resultant resin consequently having the general form shown in Fig. 1(b). The epoxy considered by Millett et al. [15], however, was more complex; its core elements were a blend of two different epoxy resins - 14-22\% 1,4butanedioldiglycidyl ether and 78-86\% an expoy based on the reaction between Bisphenol A and Epichlorohydrin (this is known as a modified Bisphenol A - Epichlorohydrin epoxy [21]). Both a hardener and flexiblazer were employed - details of both are set out in Ref. [15]. Despite all three resins considered possessing different compositions - and therefore final structures - it is notable that little difference in $U_{S}-u_{P}$ behaviour is apparent. This ties in well with the previously discussed work by Hazell et al. [14], which indicated that, at least at low pressures, $U_{S^{-}} u_{P}$ behaviour was independent of epoxy composition.

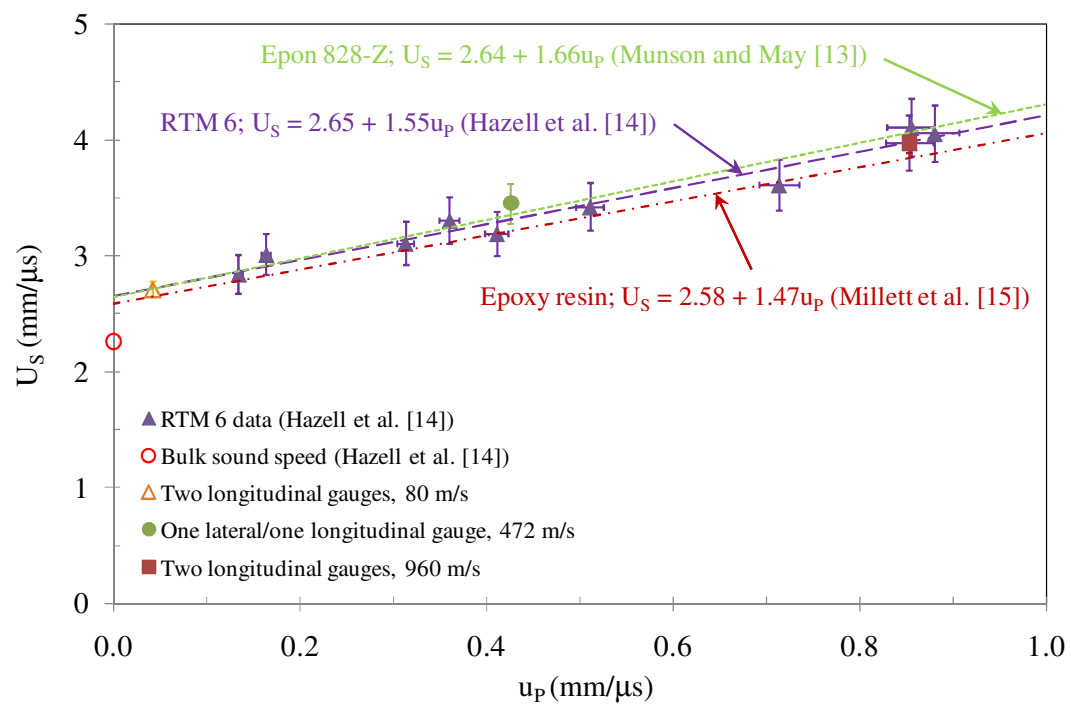

Fig. $5 U_{S^{-}} u_{P}$ Hugoniot relationships for Epon 828-Z [13], RTM 6 [14] and a bespoke epoxy resin $[15]$.

All three data points from the current work shown in Fig. 5 are a good fit with the previously derived Hugoniot. The larger error bars on the $472 \mathrm{~m} / \mathrm{s}$ shot (particularly compared to the $960 \mathrm{~m} / \mathrm{s}$ shot) arise from the uncertainty in the position of the lateral gauge used to monitor shock arrival, combined with its slower response time. The good agreement with the previously derived Hugoniot of the $960 \mathrm{~m} / \mathrm{s}$ data point is worth further comment. As this target comprised two longitudinal gauges with a centrally located lateral gauge embedded within the target material (see Fig. 4) a number of conclusions may be drawn:

1) It is possible to measure both lateral and longitudinal stresses simultaneously.

2) The good agreement with the previously derived Hugoniot implies that the centrally located encapsulated gauge packet had no material effect on the propagation of the shock through the target. The relatively sharp rise apparent in the rear surface longitudinal 
Accepted for publication in the Journal of Materials Science in August 2009; subsequently published in Vol. 44, No. 22, pp. 6187-6198 (2009).

gauge trace shown in Fig. 4 appears to confirm this analysis as no evidence of dissipation/smearing of the arriving shock is apparent.

The second point outlined above is reinforced by the high degree of impedance matching which exists between different types of epoxy [14]; this implies that an incident shock should see an effectively continuous target rather than a target face divided by a gauge encapsulation layer. In this context, it is interesting to note that the lateral gauge trace in Fig. 4 exhibits some structure behind the shock. Following an initial overshoot at shock arrival, a slight negative gradient is apparent. As discussed previously, it has been suggested that such changes in lateral stress might indicate microstructural evolution within the target material [15], although the counter-view that shock dispersion in the encapsulation region leads to the observed behaviour has also been put forward [16, 17]. The presence of this gradient, combined with the evidence from the surrounding longitudinal gauges of minimum shock dispersal, would therefore seem indicative of measurement of a change in material properties behind the shock. However, the observed gradient in lateral stress behind the incident shock is slight compared to the overall shock magnitude; further, the foil longitudinal gauges act as averaging devices over their area and the central encapsulated region of the target is relatively small compared to that area. Consequently, in order to state categorically whether or not a shock is dissipated/smeared out within the central encapsulation layer (e.g. by small differences in impedance between the different epoxy layers employed) further experiments incorporating a lateral gauge package based on an epoxy with a substantially different Hugoniot to that of the target epoxy resin would be required.

Overall, the good agreement shown in Fig. 5 between the measured $U_{S}-u_{P}$ data points from Table 2 and the known Hugoniot for RTM 6 resin gives confidence in the repeatability and consistency of this work and therefore in the lateral gauge responses measured. It should also be noted that while the Hugoniot presented in Fig. 5 has a linear nature, of the form $U_{S}=c_{0}+\mathrm{S} u_{P}$, other authors such as Porter and Gould [28] have suggested that polymers have a parabolic Hugoniot with a $U_{S}$ intercept at the zero pressure/bulk speed of sound. This is in good agreement with the low position of the measured RTM 6 bulk sound speed relative to the principle Hugoniot in Fig. 5. While there are no further significant signs of non-linearity over the particle velocity range studied, it should be stressed that the RTM 6 Hugoniot data here only represents a small range of particle velocities. It is therefore possible that at higher and lower stresses/particle velocities some non-linearity may be observed. Fig. 6 shows the $\sigma_{X}-u_{P}$ relationship for the three shots detailed in Table 2 where longitudinal stresses were measured. Quoted longitudinal stress values were averages across the Hugoniot stress plateau, with errors representing the range of data apparent within the sampling region. This data is again compared to that from Hazell et al. [14], with the hydrodynamic response based on the $U_{S}-u_{P}$ Hugoniot presented in Fig. 5 (calculated from Eq. 2, with $\rho_{0}$ as the measured density of RTM 6 from Table 1) also included for comparison. Further, the best-fit (to 
experimental data) response for the bespoke twin-epoxy (both different to RTM 6) resin studied by Millett et al. [15] is also included. Good agreement is observed between the RTM 6 data measured both here and in Ref. [14], giving further confidence in the experimental approach employed. In both cases a slight deviation above the hydrodynamic response, discussed further elsewhere, is apparent at elevated particle velocities/impact stresses. It is notable, however, that the best-fit for the epoxy resin considered in Ref. [15] shows a similar response. This is taken as further evidence of the broadly similar response of different epoxy resins, independent of composition/structure, at high strain rates previously highlighted by the similarities in the $U_{S^{-}} u_{P}$ Hugoniot equations-of-state identified in Fig. 5.

$$
P=\rho_{0} U_{S} u_{P}
$$

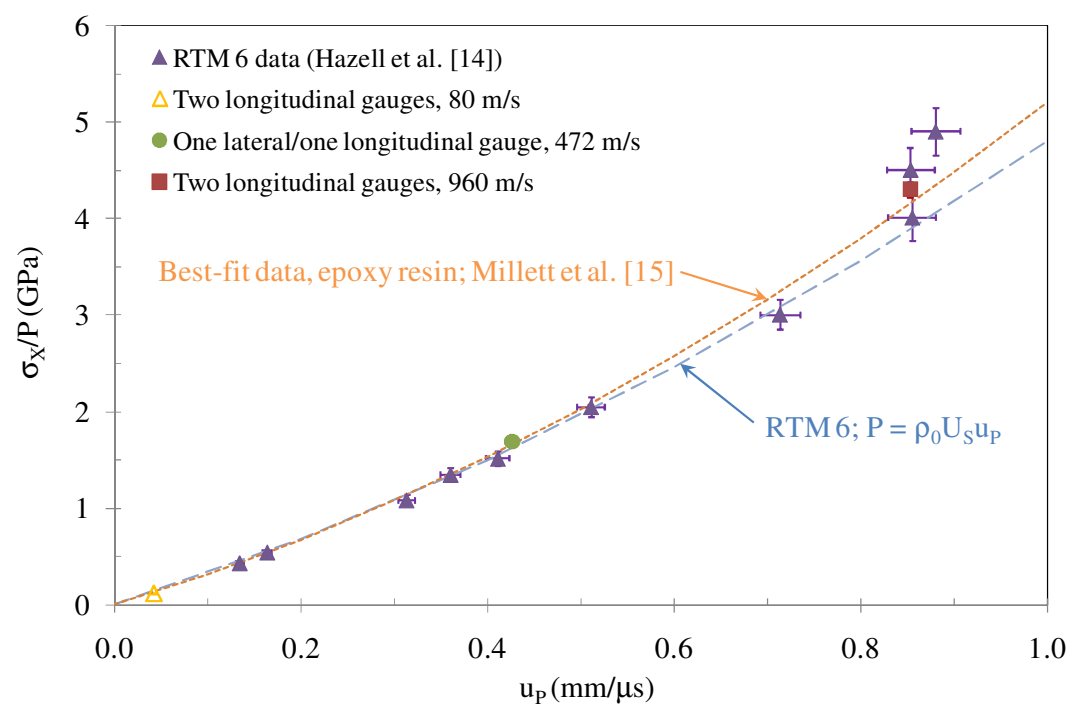

Fig. $6 \sigma_{X}-u_{P}$ Hugoniot relationships for RTM 6 [14] and a bespoke epoxy resin [15].

The longitudinal gauges employed in this work were mounted such that they directly measured the longitudinal stress. This stress consists of two components (hydrostatic and deviatoric), as shown in Eq. 3.

$$
\sigma_{X}=P+\frac{4}{3} \tau_{M A X}
$$

where $P$ is the hydrostatic pressure and $\tau_{M A X}$ is the maximum shear strength of the resin. 
It was noted from Fig. 6 that at low particle velocities/stresses the measured stresses were similar to the hydrostatic pressure, $P$. However, as observed previously by Hazell et al. [14], for $u_{P}>$ ca. $0.85 \mathrm{~mm} / \mu \mathrm{s}$, an increase in stress above the hydrodynamic pressure occurred. This implies that at these higher particle velocities/stresses there is an increase in the deviatoric component of stress - namely the maximum value of shear strength, $\tau_{M A X}$. Similar behaviour was noted by Millett and Bourne [7] in a study of three different polymers; polyethylene, polypropylene and polystyrene. An increase in the deviation from the hydrodynamic response at higher impact velocities was noted in all cases. Further, the magnitude of this increase was observed to increase with side group size/complexity, implying that the observed change in maximum shear strength was linked to microstructural changes as the impact stress increased. In essence, this effect was considered analogous to work hardening in metals, with entanglement/compression of the polymer chains leading to greater maximum shear strengths at higher impact stresses.

As outlined in Table 2, three other lateral gauge traces in addition to that presented in Fig. 4 were also recorded under a variety of impact conditions. All four traces, again with their initial rises reset to ca.0.5 $\mu \mathrm{s}$, are presented in Fig. 7. For all of the lateral gauge traces shown in Fig. 7, the time taken for the incident stress pulse to ramp up to a maximum amplitude is relatively short at $<100 \mathrm{~ns}$. The rise times are observed to increase with impact stress - with the initial gradient up to the peak stress increasing in magnitude as longitudinal stress increases. Such a relationship is apparent in the data presented elsewhere for a bespoke epoxy resin by Millett et al. [15], and is likely a function of the response of the manganin gauge to increased pressure. Further, in all of these lateral traces, but particularly for $\sigma_{X} \geq 1.69 \mathrm{GPa}$, a gradient in the measured lateral stress is apparent behind the shock before release occurs.

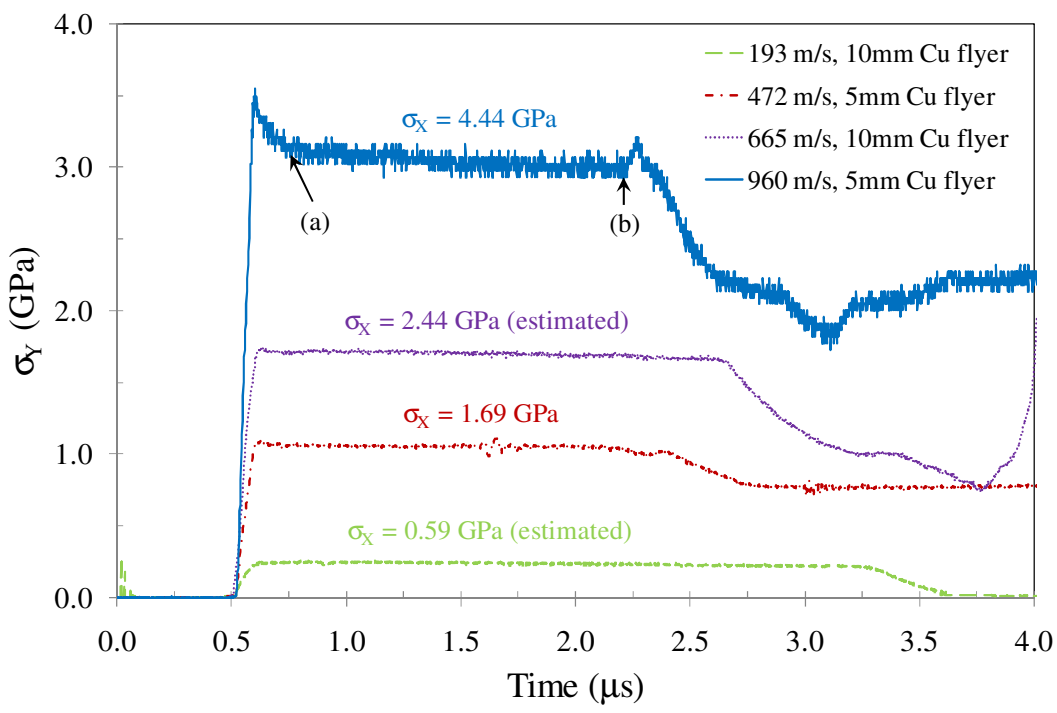


Fig. 7 Lateral stress traces following impact of Cu flyers on RTM 6 targets at 193-960 m/s.

Equation 4 relates longitudinal and lateral stresses to shear strength, $\tau$. Equation 4, unlike Eq. 3 which only deals with maximum shear strength, may therefore be used to investigate the behaviour of shear strength with time. From this equation, it is clear that if the longitudinal stress remains constant while the lateral stress decreases then shear strength will increase. As shown by Fig. 4 this is indeed the case with RTM 6 - here the longitudinal stress measured by the front surface gauge is observed to remain constant while the lateral stress decreases with time.

$$
\tau=\frac{\sigma_{X}-\sigma_{Y}}{2}
$$

While similar gradients in lateral stress behind the shock to those highlighted in Fig. 7 were observed in the epoxy resin studied by Millett et al. [15], as previously highlighted, Winter and Harris [16] have recently suggested that a modification of the interpretation of lateral gauge results, and consequently any deduced changes in shear strength, is required. The propagation of shocks through (a) a so-called matrix material, and (b) a matrix material with a central fluid layer designed to simulate an encapsulated gauge, was simulated. The presence of a fluid layer was shown to significantly modify the shock front due to different fluid layer/matrix velocities. A faster shock velocity in the fluid layer was found to result in a continual rise in lateral gauge stress after shock arrival, while a faster matrix shock front led to an initial ramp to a peak followed by a steady decrease in magnitude behind the front. The decrease in lateral stress behind the shock apparent in Fig. 7 would be consistent with the models developed by Winter and Harris [16] and Winter et al. [17] if it is assumed that the shock moves more slowly in the central encapsulation layer than in the RTM 6 matrix. However, as shown in Fig. 5/Refs. [13-15], the shock velocity (at a given particle velocity) in different epoxy resins is essentially constant. Therefore, while care must be taken in interpretation of lateral gauge traces, in this case similar shock velocities in the RTM 6/gauge encapsulation mean that dispersion of the shock is unlikely to occur. Consequently, the observed gradients in lateral stress behind the shock front in Fig. 7 are most likely a real material response rather than an artefact caused by the presence of the lateral gauge.

The change in gradient of the lateral stress behind the shock, $\Delta \sigma_{Y}$, has been measured for all four cases in Fig. 7. In each case the change in lateral stress was measured from a point on the curve after the initial response of the gauge up to a position just before the release caught up with the shock - e.g. from point (a) to point (b) for the $\sigma_{X}=4.44 \mathrm{GPa}$ case. Errors are included based on the range of possible gradients which could be measured between points (a) and (b) based on the 
scatter of the experimental data at these two sampling positions. The variation of $-\Delta \sigma_{Y}$ (used, as the gradients of the lateral stresses in Fig. 7 are negative, in order to give a positive y-axis) with $\sigma_{X}$ is plotted in Fig. 8. For comparison, this data is accompanied by results previously published by Millett et al. [15] for the previously described bespoke twin-epoxy resin.

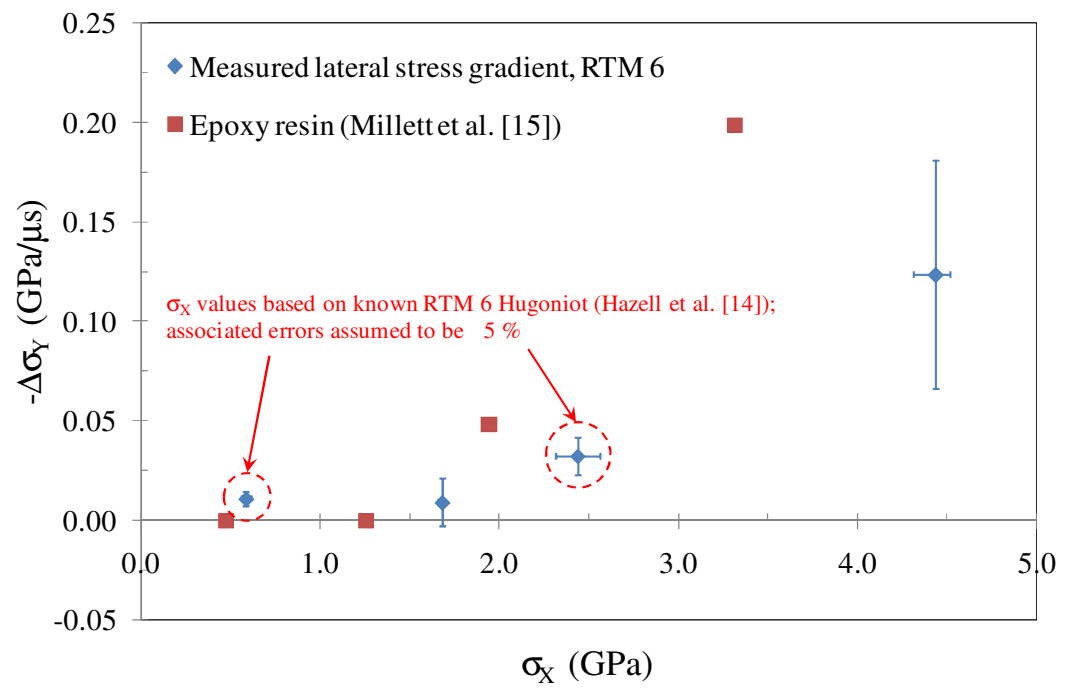

Fig. 8 Variation in lateral stress gradient with impact stress behind the main shock for a bespoke epoxy resin [15] and RTM 6 (from Fig. 7).

The aforementioned change in the lateral stress gradient, $-\Delta \sigma_{Y}$, behind the shock shown in Fig. 8 for both the experimental data and that from Millett et al. [15] is observed to increase in magnitude with impact stress. This relationship holds even when the fact that the errors in the experimental data in Fig. 8 also increase with impact stress is allowed for (this increase in the magnitude of experimental errors is a reflection of the increased noise in the higher impact stress lateral gauge traces in Fig. 7). Taking into account both the relationship between longitudinal and lateral stress and shear strength set out in Eq. 4 and the fact, already highlighted, that as shown in Fig. 4, the longitudinal Hugoniot stress tends to stay constant behind the shock, several observations may be drawn from Fig. 8: (1) up to a longitudinal impact stress of ca.1.5 GPa there is no significant change in lateral stress behind the shock - implying that no change in shear strength is occurring; (2) beyond ca.1.5 GPa a change in lateral stress occurs behind the shock, which given its magnitude implies that the resin is strengthening with time, and; (3) the magnitude of the change in lateral stress observed for $\sigma_{\mathrm{X}}>$ ca.1.5 GPa increases with impact velocity. This latter point implies that the strengthening mechanism is continuing to act up to at least $3.5 \mathrm{GPa}$. Again, this strengthening mechanism is likely to be linked to compression of the polymer chains at different impact velocities. 
It is also worth noting that, while a similar response occurs for both the epoxy resins in Fig. 8, the magnitude of $-\Delta \sigma_{Y}$ is substantially less (for a given value of $\sigma_{X}$ ) with the RTM 6 experimental data than for the different, bespoke, twin-epoxy resin from Millett et al. [15]. Lateral gauge type and the experimental technique employed were essentially identical in both sets of experiments. Further, as demonstrated here and elsewhere (e.g. Figs. 4 and 5/Ref. [14]), different epoxy resins tend to exhibit relatively similar Hugoniot equations-of-state; this means that for a given impact stress shock velocities will be broadly similar. Consequently, the difference in the shear strength behaviour for the two different resins implied by Fig. 8 suggests that lateral gauges are, at least in part, measuring an inherent physical response within the target material. Physically, this difference in material properties is most likely linked to the resin compositions. As previously set out, the epoxy resin investigated by Millett et al. [15] was a modified Bisphenol A Epichlorohydrin epoxy incorporating two different epoxies. Such modifications are designed to increase cross-linking over those resins (such as RTM 6) which only incorporate a single epoxy. Consequently, it is reasonable to attribute the enhanced strengthening behind the shock observed in this epoxy over RTM 6 to the increased resistance to compression of the polymer chains caused by increased cross-linking. This result therefore suggests that changes in lateral stress gradient behind the shock measured by manganin stress gauges are not entirely attributable to the dispersion of the incident shock by the central encapsulating layer as suggested in Refs. [16] and [17]. Instead, given the experimental evidence of near and far-field effects when a central encapsulated region is present (as demonstrated by Winter at al. [17]), a combination/superposition of two factors seems the most likely explanation for the observed lateral gauge behaviour shown in Fig. 7. These factors are: (a) physical changes in the target behind the shock, and; (b) the presence of the encapsulated stress gauge within the centre of the target acting to modify the shock front and therefore the response of the embedded gauge.

The shear strengths at the beginning/end of the shock (e.g. the locations indicated by points (a) and (b) in Fig. 7 for $\sigma_{X}=4.44 \mathrm{GPa}$ ), calculated from the data presented in Table 2 according to Eq. 4 are plotted against longitudinal impact stress in Fig. 9 (an approach previously employed elsewhere, e.g. [27, 29]). It is worth noting that the average shear strength would, in each case, lie approximately halfway between these two values. Errors in shear strength were derived from the experimental errors in $\sigma_{X}$ and $\sigma_{Y}$ - with errors in the $\sigma_{X}$ components assumed to be $\pm 5 \%$ where no longitudinal gauge was employed (e.g. where a value based on the Hugoniot relationship presented in Fig. 6 was used). The predicted isotropic elastic response of RTM 6 based on Eq. 5 is also included $[15,27,29]$. This elastic response depends on both the Poisson's ratio $v$ and the impact stress. Further, average shear strength data derived in the same manner (e.g. according to Eq. 4) for the bespoke two-element epoxy considered by Millett et al. in Ref. [15] have been included in order to provide a point of comparison. 


$$
2 \tau=\frac{1-2 v}{1-v} \sigma_{x}
$$

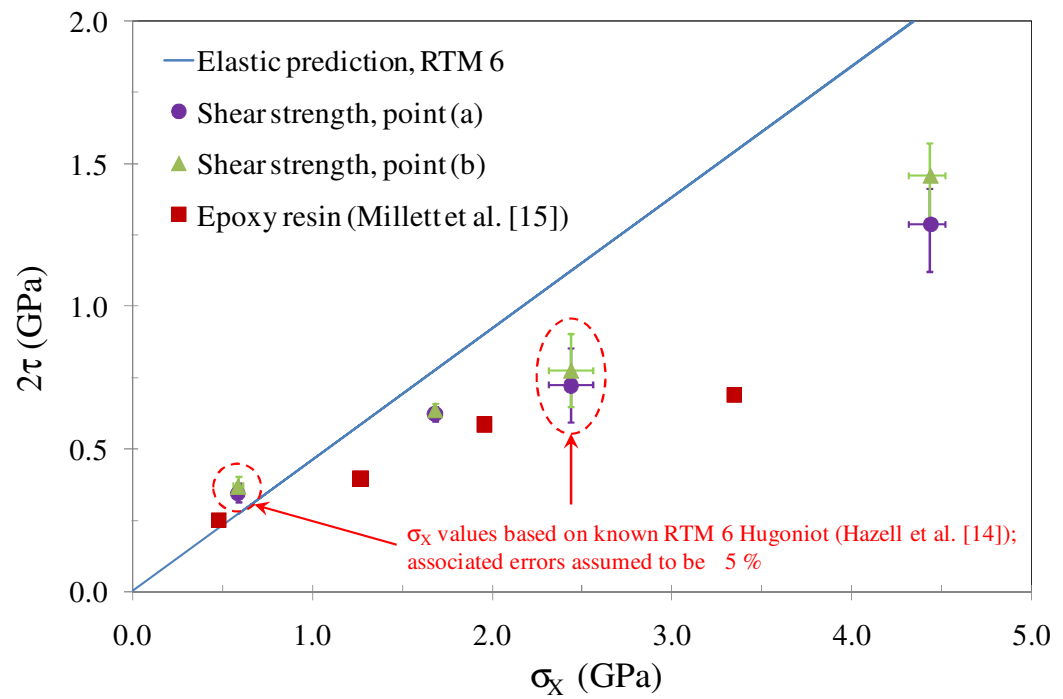

Fig. 9 The variation in shear strength with impact stress for RTM 6 at the positions highlighted in Fig. 7.

Shear strength is observed to increase with impact stress. The gradual movement of the data points from locations (a) and (b) in Fig. 7 below the elastic prediction with increasing stress indicates that at higher impact stresses a plastic deformation mechanism is active. It is notable that the data from Ref. [15] falls below both sets of data for RTM 6. This is in good agreement with the observed greater gradient in lateral stress behind the shock illustrated in Fig. 8 and implies that the twin-epoxy bespoke resin employed by Millett et al. [15] undergoes greater strengthening behind the shock then RTM 6. As before, this is most likely a result of the greater cross-linking (and therefore resistance to compression) which will be present in a twin versus single epoxy resin system. As the movement of data below the elastic prediction represents plastic deformation, it follows that if a line of best fit were passed through the two sets of data from locations (a) and (b), the Hugoniot elastic limit (HEL) of RTM 6 should be represented by the point of departure of these lines from the elastic response. While not included for clarity, best-fit lines through the data from points (a) and (b) would intercept the elastic prediction at $\sigma_{X}$ values of 0.84 and $0.91 \mathrm{GPa}$ and $2 \tau$ values of 0.39 and $0.42 \mathrm{GPa}$ respectively. This would give an average HEL of $0.88 \pm 0.04 \mathrm{GPa}$. The fact that the RTM 6 data point/associated error bars for $\sigma_{X}=0.59 \mathrm{GPa}$ lie just above the elastic response should also be highlighted. This is likely attributable to the requirement to estimate both $\sigma_{X}$ and the associated errors in this case (see Table 2). As shown in Fig. 6, the $\sigma_{X}-u_{P}$ Hugoniot is ill-defined at lower impact stresses; consequently, it might be expected that the errors 
on estimated values of $\sigma_{X}$ would be substantially greater at lower stresses. Nevertheless, the broadly linear variation in shear strength with impact stress shown in Fig. 9 gives confidence in the overall validity of this approach. For comparison, Millett et al. [15] calculated the HEL of a bespoke epoxy resin using the same technique. They found a value of $\sim 0.6 \mathrm{GPa}$ and, additionally, by reviewing previous work by Barker and Hollenbach [6], suggested a HEL for PMMA of $~ 0.9$ GPa. This value was of the same order of magnitude as that measured by Barker and Hollenback [6] themselves (of $0.7 \mathrm{GPa}$ ) for PMMA. This result implies that the method used here, and therefore the HEL of ca.0.88 GPa derived in this work for RTM 6, is also of the correct order of magnitude. Additionally, the tendency of the points sampled at location (b) in Fig. 7 to stray below those from location (a) with increasing impact stress in Fig. 9 represents further evidence of the previously discussed increase in shear strength behind the shock. However, in all cases the error bars for the data points from both locations overlap meaning that there is insufficient spatial separation of the data points to quantify this effect.

\section{Conclusions}

The dynamic response of the commercially important RTM 6 resin has been investigated using plate impact experiments in the impact velocity regime $80-960 \mathrm{~m} / \mathrm{s}$. Both longitudinal and lateral gauges were employed and results were compared to the literature. A linear $U_{S^{-}} u_{P}$ Hugoniot was confirmed, in line with data from the literature for RTM 6, with no evidence of any non-linearity at lower particle velocities as observed elsewhere for the thermoplastic PMMA. The derived $\sigma_{X^{-}}$ $u_{P}$ Hugoniot evidenced an increase in longitudinal stress above the hydrodynamic response at elevated particle velocities. This was interpreted as an increase in maximum shear strength as the impact velocity increased, attributed to increased resistance to compression due to polymer chain compression. With both the $U_{S^{-}} u_{P}$ and $\sigma_{X}-u_{P}$ Hugoniot relationships, comparison to data from the literature showed that the equations-of-state were similar for epoxy resins with different compositions; a result expected based on previous work by some of the authors of this paper.

Lateral gauge traces showed a fast rise, followed by an overshoot and than a plateau before releases from the flyer plate ended the 1D behaviour at the gauge. A gradient in the recorded lateral stress histories following shock arrival was apparent for $\sigma_{X}>1.5 \mathrm{GPa}$ and was tentatively interpreted as an increase in shear strength behind the shock. While small, this effect appeared measurable and consequently implied that a hardening mechanism - again likely linked to polymer chain compression - was in operation behind the shock for impact stresses $>1.5 \mathrm{GPa}$. Comparison of the magnitude of the lateral stress gradient behind the shock in RTM 6 for different impact stresses was made to data for a bespoke twin-epoxy resin from the literature. Despite the similar $U_{S^{-}} u_{P}$ Hugoniot relationships, these two different resins showed substantial differences in the magnitude of the gradient in lateral stress behind the shock. For example, for impact stresses of 
Accepted for publication in the Journal of Materials Science in August 2009; subsequently published in Vol. 44, No. 22, pp. 6187-6198 (2009).

3.0-4.5 GPa, a >100 \% difference in lateral gradient magnitude was apparent. A similar (but less marked) difference was also apparent when the variation of shear strength with impact stress was considered. Shear strengths within RTM 6 were determined by comparing recorded/calculated longitudinal and lateral stresses. The embedded lateral gauges allowed direct monitoring of changes in shear strength behind the shock. By calculating shear strengths at both the beginning and end of lateral stress plateaus it proved possible to quantify the observed increase in shear strength in the region behind the shock. Comparison of these results was again made to similarly derived data for a bespoke twin-epoxy resin from the literature (in this case with lateral stresses just taken from a point immediately following shock arrival rather than two locations). In both cases, maximum shear strength was observed to increase in magnitude with impact velocity. This confirmed the relationship suggested by both the derived RTM 6 and literature-based twin-epoxy $\sigma_{X}-u_{P}$ Hugoniot's, where $\sigma_{X}$ was observed to trend above the predicted elastic response at higher particle velocities. This behaviour was attributed to a mechanism analogous to "hardening" in metals involving increased entanglement/compression of the polymer chains at higher impact stresses leading to greater resistance to subsequent compression. The difference in both lateral stress gradient behind the shock/variation of shear strength with impact stress for RTM 6 and the twin-epoxy resin considered from the literature was attributed to the different chemical structures of the two epoxies. The twin-epoxy exhibited a larger gradient in lateral stress behind the incident shock front/greater strengthening then the RTM 6, presumably due to greater internal cross-linking leading to enhanced resistance to compression. Essentially, it is suggested that the same polymerchain-compression mechanism identified above also accounts for the difference in behaviour between these two resins.

Recent work has suggested that the response of lateral gauges behind the shock is, at least initially, attributable to the composite nature of the gauge package/target assembly, with the smearing of the incident shock across both elements leading to the observed changes in gradient. However, given the otherwise similar nature of the two epoxy resins considered here (particularly the similar $U_{S^{-}} u_{P}$ Hugoniot relationships, which imply similar shock velocities for a given impact stress), the observed differences in behaviour behind the shock were taken to imply that the lateral gauges were detecting changes in a physical material property within the target material. Additionally, the results of a test undertaken with both a central embedded lateral gauge and front/rear longitudinal gauges (where no evidence of shock modification was noted at the rear surface) appeared to provide further evidence that shock dispersal in the encapsulated lateral gauge package was minimal. While the significance of this result was initially qualified, when considered in light of the different lateral gauge responses in otherwise similar resins, it appears to back the conclusion that embedded lateral gauges are picking up some kind of material response. Consequently, this work suggests that in epoxies observed changes in lateral manganin gauge response behind the shock front may be primarily attributed to a change in material properties. 
Shear strength data also allowed determination of the HEL of Hexcel ${ }^{\circledR}$ RTM 6; the intercepts between the two experimental data sets and the elastic best-fit yielded an estimate of $0.88 \pm 0.04$ GPa. This result was of a similar order of magnitude to data from the literature for both PMMA and a twin-epoxy resin system which demonstrated similar $U_{S}-u_{P} / \sigma_{X}-u_{P}$ Hugoniot responses.

Overall, knowledge of the dynamic response of the important epoxy resin Hexcel ${ }^{\circledast}$ RTM 6 has been extended to regimes/strain rates beyond the data previously available in the literature. Evidence of strengthening both with increased impact stress and behind the shock has been found and tentatively linked to resistance to compression of polymer chains. By comparison to data from the literature, this result has been used to enhance the validity of the embedded lateral gauge technique with regards to the measurement of physical target material properties behind the shock.

However, further work will be required to determine the extent of any target/gauge package geometrical effects on embedded lateral gauge response. Good agreement between the derived Hugoniot relationships and the identified RTM 6 HEL with data for similar polymers from the literature creates a reasonable degree of confidence in the validity of these results.

\section{Acknowledgements}

The authors wish to thank Mr Steven Mortimer of Hexcel, Duxford, UK, for supplying the cured resin samples. In addition, the authors gratefully acknowledge the invaluable aid of $\mathrm{Mr}$ Gary Cooper in carrying out this work.

\section{References}

[1] Millett JCF, Bourne NK, Meziere YJE, Vignjevic R, Lukyanov A (2007) Composites Sci. Technol. 67:3253-3260

[2] Ryan S, Schfer F, Guyot M, Hiermaier S, Lambert M (2008) Int. J. Impact Engng. 35:17561763

[3] Meyers MA (1994) Dynamic Behavior of Materials. John Wiley \& Sons, Inc.

[4] Field JE, Walley SM, Proud, WG, Goldrein HT, Siviour CR (2004) Int. J. Impact Engng. 30: $725: 775$

[5] Millett JCF, Bourne NK, Gray III GT, Cooper G (2001) Shock Compression of Condensed Matter CP620:131-134

[6] Barker LM, Hollenbach RE (1970) J. Appl. Phys. 41, no.10:4208-4226

[7] Millett JCF, Bourne NK (2004) J. Phys. D: Appl. Phys. 37:2901-2907

[8] Hazell PJ, Edwards MR, Longstaff H, Erskine J (2009) Int. J. Impact Engng. 36:147-153 
Accepted for publication in the Journal of Materials Science in August 2009; subsequently published in Vol. 44, No. 22, pp. 6187-6198 (2009).

[9] Bourne NK, Millett JCF (2003) Proc. R. Soc. Lon. A 459:567-576

[10] Marsh S P (1980) LASL Shock Hugoniot Data. University of California Press, Ltd.

[11] Gerlach R, Siviour CR, Petrinic N, Wiegand J (2008) Polymer 49:2728-2737

[12] Hazell PJ, Stennett C, Cooper G (2009) Composites: Part A 40:204-209

[13] Munson DE, May RP (1972) J. Appl. Phys. 43, no.3:962-971

[14] Hazell PJ, Stennett C, Cooper G (2008) Polymer Composites 29, no.10:1106-1110

[15] Millett JCF, Bourne NK, Barnes NR (2002) J. Appl. Phys. 92, no.11:6590-6594

[16] Winter RE, Harris EJ (2008) J. Phys. D: Appl. Phys. 41:035503

[17] Winter RE, Owen GD, Harris EJ (2008) J. Phys. D: Appl. Phys. 41:202006

[18] Skordos AA, Karkanas PI, Partridge IK (2000) Meas. Sci. Technol. 11: 25:31

[19] Aduriz XA, Lupi C, Boyard N, Bailleul J-L, Leduc D, Sobotka V, Lefèvre N, Chapeleau X, Boisrobert C, Delaunay D (2007) Composites Sci. Technol. 67: 3196:3201

[20] HexFlow ${ }^{\circledR}$ RTM $6180^{\circ} \mathrm{C}$ epoxy system for Resin Transfer Moulding monocomponent system: Product Data. Hexcel Composites (Duxford UK):

http://www.hexcel.com/Products/Downloads/RTM\%20Data\%20Sheets/

[21] Saunders KJ (1988) Organic Polymer Chemistry, Second Edition. Chapman \& Hall, London

[22] Safety Data Sheet RTM6. Hexcel Composites (Duxford UK)

[23] Bourne NK (2003) Meas. Sci. Technol. 14:273-278

[24] Rosenburg Z, Partom Y (1985) J. Appl. Phys. 58, no.8:3072-3076

[25] Rosenburg Z, Brar NS (1995) J. Appl. Phys. 77, no.4: 1143-1148

[26] Millett JCF, Bourne NK, Rosenberg Z (1996) J. Phys. D: Appl. Phys. 29:2466-2472

[27] Appleby-Thomas GJ, Hazell PJ, Stennett C, Cooper G, Helaar K, Diederen AM (2009) J.

Appl. Phys. 106: 064916-1-064916-9

[28] Porter D, Gould PJ (2006) J. Phys. IV France 134:373-378

[29] Millett JCF, Bourne NK, Dandekar DP (2004) J. Appl. Phys. 96, no.7:3727-3732 